\title{
MAFLD-Epidemiology, Natural History, Outcomes and Prevention
}

\author{
Aimun Raees, Muhammad Kamran, and Wasim Jafri
}

\section{ABSTRACT}

Scientists have recently modified the term Non-Alcoholic Fatty Liver Disease (NAFLD) to Metabolic Dysfunction-Associated Fatty Liver Disease (MAFLD) in an attempt to improve the understanding and overall outcomes of the disease. The leaping prevalence and formidable mortality rate of fatty liver disease throughout the world is quite worrisome. Due to the lack of appropriate knowledge of the natural history of disease, suitable pharmacotherapy could never be devised. Thus, the management solely relies on patients' earnest co-operation with fierce lifestyle changes such as exercise, weight loss and dietary modifications. In this era, it is essential to come up with strategies to curtail the underlying risk factors in order to prevent the rapid progression of MAFLD. In this review, we will discuss the epidemiology of NAFLD and the newly found evidence on prevalence of MAFLD as well as the disease outcomes and preventive measures.

Keywords: Epidemiology, MAFLD, NAFLD, Natural history, Prevention.
Submitted : Jun 20, 2021

Published : July 20, 2021

ISSN: 2593-8339

DOI: $10.24018 /$ ejmed.2021.3.4.941

Aimun Raees

The Aga Khan University Hospital, Karach Pakistan.

(e-mail: aimunraees@ gmail.com)

Muhammad Kamran*

Fazaia Ruth Pfau Medical College, Karach Pakistan.

(e-mail: muhammadkamran81 @ gmail.com) Wasim Jafri

The Aga Khan University Hospital, Karach Pakistan.

(e-mail: wasim.jafri@aku.edu)

*Corresponding Author

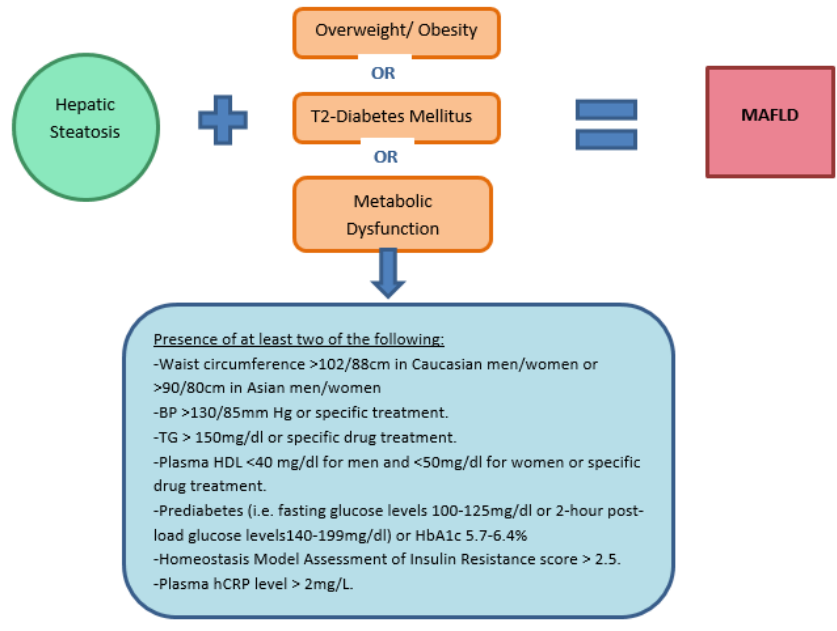

Fig. 1. Diagnostic criteria of MAFLD.

\section{EPIDEMIOLOGY}

MAFLD is a multi-system disease that stems from interaction between metabolic, environmental, and behavioral factors on the background of genetic predisposition. The data on MAFLD alone is scarce as its still under the process of being accepted as an updated term for NAFLD. Globally, owing to the soaring pandemic of obesity and type-2 Diabetes Mellitus (T2DM), MAFLD has swiftly become the leading cause of end stage liver disease in all age groups along with hepatocellular carcinoma (HCC) and an 
indication of liver transplant [2], [3]. As of now, approximately $25 \%$ of the world's population is estimated to have NAFLD but its prevalence greatly varies according to regional differences as well as exposure to risk factors. Overall, prevalence is noted to be highest in Middle East, while in Asia, NAFLD seems to be the most prevalent in China [4].

Table I shows region wise prevalence of NAFLD in descending order.

TABLE I: REGIONAL PREVALENCE OF NAFLD

\begin{tabular}{ccc}
\hline S. No. & Region & Prevalence \\
\hline 1 & Middle East & $31.79 \%[3]$ \\
2 & South America & $30.45 \%[3]$ \\
3 & China & $36.83 \%[5]$ \\
4 & Asia & $27 \%[4]$ \\
5 & North America & $24.13 \%[3]$ \\
6 & Europe & $20-23.7 \%[3]$ \\
7 & UK & $20.7 \%[6]$ \\
8 & Africa & $13.48 \%[3]$ \\
\hline
\end{tabular}

Table II shows prevalence of NAFLD according to associated risk factors.

TABLE II: NAFLD PREVALENCE IN VARIOUS GROUPS AT RISK

\begin{tabular}{ccc}
\hline S. No. & Population At Risk & Prevalence \\
\hline 1 & Morbid Obsecity & $95 \%[7]$ \\
2 & Hyperlipidemia & $90 \%[8]$ \\
3 & T2DM & $57.80 \%[9]$ \\
4 & Children & $3-10 \%[10]$ \\
5 & Obese Children & $40-70 \%[10]$ \\
\hline
\end{tabular}

According to a recent meta-analysis, the global prevalence of lean NAFLD is $9.7 \%$ [5]. Exact prevalence of NonAlcoholic Steato-hepatitis (NASH) is unknown due to lack of histological data. However, estimated prevalence of NASH in overall population ranges from $1.5-6.45 \%$ [6]. Among morbidly obese patients going through bariatric surgery, NAFLD prevails in over $95 \%$ whereas $20-50 \%$ are found to have NASH and $10 \%$ have fibrosis [7]. As per the National Health and Nutrition Examination Survey (NHANES), the prevalence of MAFLD in the United States from 2011 to 2018 was $34.8 \%$ with a higher percentage in men compared to women $(38.5 \%$ versus $31.1 \%)$. MAFLD was more prevalent in older age, increasing from $23.2 \%$ for age $18-39$ years to $43.8 \%$ in individuals older than 60 years. Concomitant liver disease was found in $7.6 \%$ patients that included $0.5 \%$ chronic hepatitis- $\mathrm{B}, 1.6 \%$ chronic hepatitis-C and $5.5 \%$ alcoholic liver disease [8]. The prevalence of MAFLD in Chinese population was found to be $26.1 \%$ [9]. Overall, the prevalence of MAFLD is predicted to shoot up exponentially in the coming years due to the increase in metabolic disorders alongside sedentary lifestyle. A recent study that used Markov model has demonstrated that the incidence of decompensated cirrhosis is expected to rise $168 \%$ by 2030 while that of HCC will increase by $137 \%$ [10].

\section{NATURAL HISTORY AND OUTCOMES OF MAFLD}

The true course of fatty liver disease is obscure, due to paucity of evidence on histologically proven disease and high risk of selection bias encountered while performing analysis of patients with liver biopsies. Nevertheless, NAFLD is considered a slowly progressive disorder that may not always result in end-stage liver disease. It evolves from steatosis without hepatitis to non-alcoholic steatohepatitis (NASH) that may eventually turn in to advanced fibrosis with associated hepatic and extra hepatic complications.

Generally, the histologic progression from one stage of fibrosis to another takes about 14.4 years in NAFLD and approximately 7 years in NASH [11]. Studies have shown that about $10-30 \%$ of patients with NAFLD may progress to NASH and out of those, 10-15\% may develop cirrhosis over a period of 10-20 years [12]. Silent cirrhosis is revealed in about $10 \%$ patients on histological analysis. In decompensated cirrhosis, the prognosis is indistinguishable from other causes of chronic liver disease [13]. Risk of developing HCC is not very high in patients with NAFLD, about $0.44 / 1000$ person years whereas among individuals with NASH, the incidence of HCC jumps up to 10-fold, estimating 5.29/1000 person years [14], ranging from $2.4 \%$ over 7 years to $12.8 \%$ over 3 years [15].

Fig. 2. represents the natural history of the fatty liver disease.

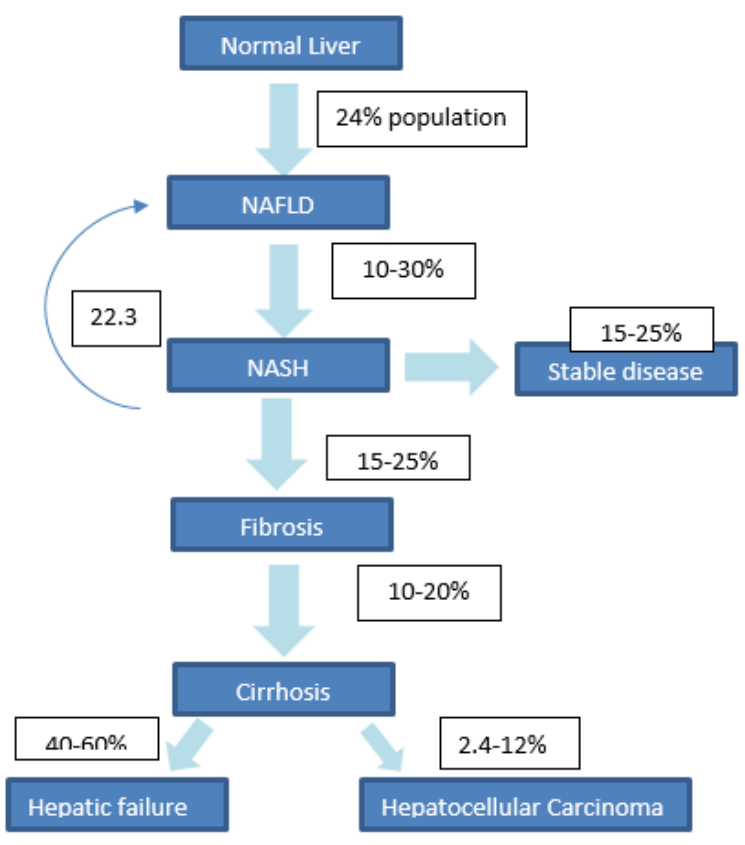

Fig. 2. Depiction of natural history of fatty liver disease.

Besides metabolic factors, aging, race, ethnicity, genetic and environmental risk factors have also been implicated in the disease process [16]. A synergistic effect between all of these mediates the overall progression of the disease. Knowledge and identification of these risk factors is important as it permits a personalized approach to advocate preventive strategies or pharmacotherapy as progression of MAFLD is subjected to alterations in these risk factors.

Studies have emphasized that visceral obesity, rather than BMI alone, is a more accurate predictor of severity of NAFLD. Dysfunctional and hypertrophied adipocytes activate macrophages and increase level of inflammatory cytokines such as interleukin- 6 and Tissue necrosis factorAlpha, directly affecting insulin resistance, which eventually leads to worsening of steatosis. The importance of visceral adipocyte accumulation can also be proved by the entity lean NAFLD, where patients with low BMI develop insulin resistance and liver damage due to high visceral adipose 
tissue content and associated lipotoxicity and insulin resistance [17], [18]. According to a study, NAFLD was reported in 3-30\% of non-Obese individuals, especially among Asian population [19]. The significance of obesity in the pathogenesis of disease progression also supports the diagnostic criteria for MAFLD. The estimated global burden of MAFLD among obese adults to reported to be as high as $51.3 \%$ in a recent meta-analysis [20].

Insulin resistance is directly proportional to the severity of histological lesions as well as fibrosis progression. It also serves as a potent link between T2DM and NAFLD. According to data, NAFLD is found in about $75 \%$ of diabetic patients while $25 \%$ of patients with NAFLD have T2DM [21]. The risk of hospitalization also escalates up to 3-5 folds in diabetic patients who have chronic liver disease secondary to NAFLD. The probability of HCC development is 2-4 times higher in patients with T2DM irrespective of other risk factors [22]. Consequently, screening of NAFLD in T2DM and vice versa was recommended [23]. As insulin resistance is one of the key driving factors for hepatic fat accumulation, therefore it has rightly been added as a major criterion for diagnosing MAFLD.

Emerging data has proved that environmental and genetic risk factors are as crucial as clinical factors in predicting fibrosis progression. According to the third NHANES study [24], Hispanics were found to have a significantly higher risk of NAFLD development while as per inter-ethnic studies, the risk was intermediate in Europeans and lower in Africans, independent of confounders [25]. Genetic polymorphism has also been highlighted as a determinant of susceptibility to MAFLD as well as steatosis severity. The most critical one is the palatin-like phospholipase domain-containing 3 (PNPLA3) gene, that accounts for a higher risk of disease in Hispanic population [26]. It has been described as an independent cause of hepatic steatosis irrespective of metabolic risk factors [27]. Early hepatic decompensation and development of HCC has also been linked to the homozygosity of this mutation [28], [29]. Other mutations responsible for regulating hepatic lipid content include Transmembrane 6 superfamily member-2 (TM6SF2) [30], Membrane bound O-Acyl Transferase 7 (MBOAT7) [31], Glucokinase regulator (GCKR) [32], LIPA gene [33], 17-beta hydroxysteroid dehydrogenase-13 (HSDL7B13) [34] and Protein phosphatase 1 regulatory subunit 3b (PPR1R3B) [35]. The last two serve as protective mutations while PNPLA3, TM6SF2 and MBOAT7 have a crucial part in amplifying the impact of MAFLD on hepatic outcomes [36]. Arterial hypertension, sarcopenia and environmental toxins exposure have also been related to worse disease outcomes.

Growing evidence suggests that NAFLD is a heritable disorder as the chances of progression seem to be greater in first degree relatives of NAFLD related chronic liver disease in comparison to general population, irrespective of risk factors [37]. The role of epigenetic changes is under experiment as few studies have displayed a positive correlation between disturbances in fetal and infancy and acquiring NAFLD in later life [38], [39]. Dysregulation of micro-RNA (mi-RNA) was shown to have an impact on both hepatic steatosis and fibrosis. [40]. Imbalance in gut microbiota may increase susceptibility to NALFD by enhancing free fatty acids absorption and increased bacterial permeability in small bowl, which in turn alters fat and glucose metabolism by generating inflammatory cytokines and activating Farnesoid X-receptors [41].

Fig. 3. depicts the associated risk factors and outcomes of MAFLD.

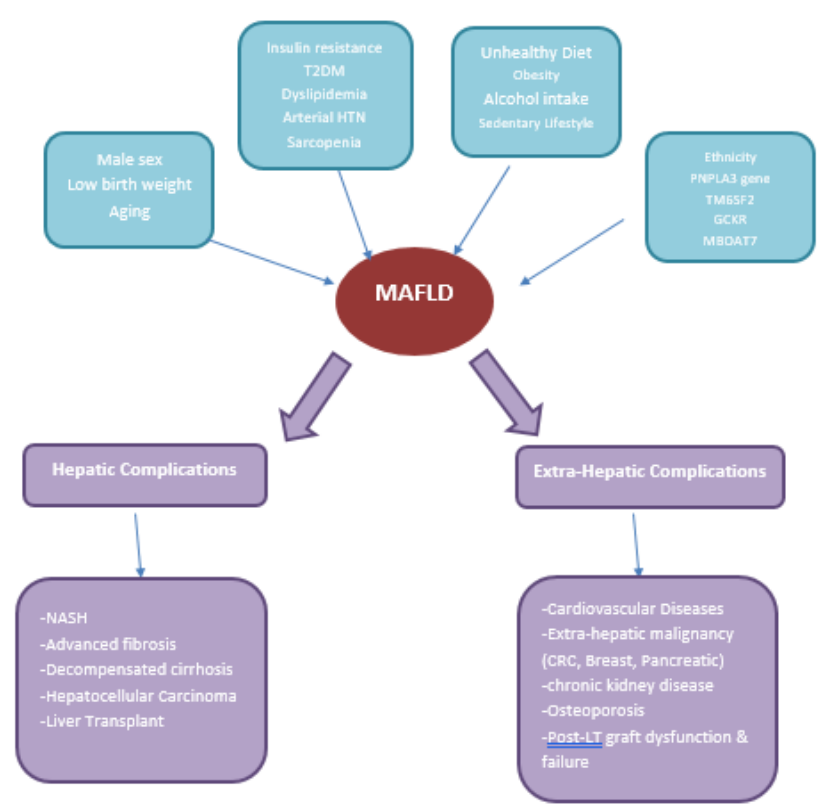

Fig. 3. Representation of risk factors and outcomes of MAFLD.

\section{MORTALITY}

The rate and cause of mortality is greatly variable according to the stage of fibrosis. NAFLD alone may not have a great impact on mortality rate, but the prognosis differs as it transitions into subsequent stages. Regardless, patients with fibrosis always have a poor survival than patients without fibrosis [42]. According to a recent meta-analysis, the estimated all-cause mortality rate ratio for stage-I fibrosis was 1.58, worsening in accordance to fibrosis stages $2,3,4$ to $2.25,3.48$ and 6.40, respectively [43]. Due to the identical predisposing factors, a strong correlation is found between fatty liver disease and cardiovascular disorders. The cumulative incidence of all-cause mortality was higher in MAFLD-group (26.2\%) than in NAFLD-only group (10.6\%) [44]. Among patients with NAFLD without or early NASH, cardiovascular events are seen to be the most frequent cause of death followed by extra-hepatic cancers and then liver related events [45]. Previously, a meta-analysis by Targher et al [46] had reported an odds ratio of 1.31 for fatal cardiovascular diseases in patients with NAFLD. Recently, in the largest nationwide cohort study done in Korea, Lee et al evaluated the incidence of cardiovascular adverse effects by using definitions of both NAFLD and MAFLD. Besides finding a higher prevalence of MAFLD, it was also noted that the hazard ratio for cardiovascular events escalated stepwise from 1.09 in the NAFLD group to 1.43 in the MAFLD group [47]. Another extra-hepatic outcome of MAFLD is the growing numbers of chronic kidney disease (CKD) among this group, that poses a negative effect on the overall prognosis of a patient [48]. NHANES 2017-2018 highlighted that the chances of developing CKD in MAFLD patients were much higher although there was no independent association 
between the two. A recent cross-sectional study done on the data from NHANES-III revealed a considerably greater prevalence of CKD in individuals with MAFLD when compared to NAFLD group (29.60\% versus $26.56 \%$ ) [49].

Patients who have developed fibrosis, liver-related mortality is more predominant with an estimated rate of 11.77/1000 person years [50], [51]. Besides mortality, NAFLD-related cirrhosis and HCC have also become one of the most leading causes of liver transplantation in the West. Over the past decade, the need of liver transplant due to NAFLD has risen by $170 \%$ [52], [53]. According to a recent cohort study, the incidence of diabetic patients requiring liver transplant for decompensated cirrhosis due to NAFLD was $305 / 100,000$ person years [54]. Contrary to the common belief that HCC is not very common in fatty liver disease, recent data reported a surge in the burden of HCC secondary to MAFLD [55]. HCC essentially contributes to death as demonstrated by a substantial rise in HCC-related mortality rate for NAFLD (19.1\%) [56].

\section{PREVENTION}

Histological staging and fibrosis are the determinants of prognosis, in association with the metabolic factors. Management of the disease is thus aimed at slowing the progression of disease by altering the modifiable risk factors.

All the scientific societies have concurred that weight loss is the most effective step in modifying disease process. Therefore, the recommended first line measure against fatty liver disease is physical activity along with consumption of a healthy diet [57]. Long term follow-up studies have demonstrated a positive effect of weight loss on both histological activity and fibrosis scores [58]. A dosedependent response is observed between the amount of weight loss and change in histological stage. A 5\% weight loss has been linked [59] with steatosis improvement while $10 \%$ reduction in weight leads to fibrosis regression [60]. In another prospective study, reduction in BMI and waist circumference were identified as independent factors for fibrosis regression and stable liver disease activity, noted in $25 \%$ and $48 \%$ of patients, respectively [61]. Bariartic surgery aids in accelerated weight loss and has been put forward as a suitable therapeutic modality, due to its favorable effect on liver histology. However, some studies also showed worsening of fibrosis [62]. The data available is not enough to draw conclusions and randomized control trials are needed to validate the true outcomes of bariatric surgeries. Endoscopic placement of intra-gastric balloons (IGB) is another less-invasive intervention that assists in weight reduction. Lately, a meta-analysis done on the patients with MAFLD undergoing IGB placement demonstrated significant improvement in the metabolic parameters of the disease. It is imperative to note that the merits of weight loss have been observed in both obese and lean patients [63].

The quality of diet plays a pivotal role in the development of MAFLD. Consumption of diet rich in saturated fats and fructose and poor in polyunsaturated fatty acids, fiber and antioxidants have deleterious effects on health. High glycemic index foods cause prolonged hyperglycemia and increase circulating insulin levels thus enhancing lipogenesis and hepatic fat accumulation [64]. Fiber deficiency in diet may alter gut microbiota eventually adding to hepatic fat burden [65]. Therefore, a high fiber diet, with low fat and carbohydrate content is recommended. To achieve that, Mediterranean diet has been proposed as a healthy eating choice. Increased consumption of vegetables, fruits, nuts, whole-grains, legumes and fish and low intake of red meat and alcohol are the characteristics of Mediterranean diet. A randomized study indicated that liver fat was relatively reduced by $40 \%$ in patients who consumed Mediterranean diet [66].

Physical activity itself, irrespective of weight loss, has illustrated beneficial effects on hepatic steatosis [67]. Exercise increases hepatic clearance of insulin and reduced its secretion from pancreatic cells and also activates AMPK5 (5'-Adenosine Monophosphate-activated Protein Kinase). Aerobic exercise is especially linked with diminished visceral obesity thus improving histological endpoints in MAFLD [68]. No predefined criteria exist regarding duration or intensity of workout required to produce favorable outcomes, but vigorous exercise alone was able to induce a notable reduction in NASH [69]. Combination of diet restriction and exercise has a more remarkable outcome than either of these alone.

Achieving these intense lifestyle modifications is quite hard and sustaining-even harder, hence a compulsion to devise pharmacotherapy arises for at least the progressive form of disease. Several drugs based on the commonly associated metabolic factors have been thoroughly investigated, from lipid lowering agents to hypoglycemic drugs, ursodeoxycholic acid and probiotics but none of these have had a remarkable effect in altering the course of disease. Vitamin-E based on its antioxidant properties succeeded in reducing hepatic steatosis but failed to have an impact on fibrosis. Nevertheless, it has been recommended for an offlabel use, in patients with NASH without T2DM [70], [71]. Recently, a Farnesoid-X receptor agonist, Obeticholic acid, has demonstrated promising results in the improvement of both hepatic steatosis and fibrosis, confirmed via histological analysis [72]. FDA approval of this drug is eagerly awaited.

\section{CONCLUSION}

In essence, the burden of NAFLD is overwhelming. The prevalence of the disease may increase even further once the diagnostic criteria of MAFLD will be put into use fur future studies. As per a recent study, MAFLD was able to capture more patients accurately in comparison to NAFLD, having a direct impact on the overall prevalence of the disease [73]. It is unfortunate that the disease that was once considered to be benign is currently on a trajectory to be the most leading etiology of decompensated cirrhosis, hepatocellular carcinoma, and liver transplant worldwide. The morbidity and mortality associated with extra-hepatic components such as cardiovascular events and extra-hepatic malignancies is also alarming. The heterogeneity observed in the course of the disease makes it even more challenging and incommodious. Therefore, as long as target pharmacotherapy is not available, vigilant screening and early preventive measures are required to curb the disease before it blooms. Awareness regarding the nature of disease and counseling sessions to emphasize on the vigorous life-style modifications 
are mandatory. Further research is crucial to improve the understanding of the disease in order to provide better therapeutic strategies.

\section{REFERENCES}

[1] Valenti, Gamal Shiha, Claudio Tiribelli, Hannele Yki-Järvinen, JianGao Fan, Henning Grønbæk, Yusuf Yilmaz, Helena Cortez-Pinto et al. "A new definition for metabolic dysfunction-associated fatty liver disease: an international expert consensus statement".

[2] Wong RJ, Aguilar M, Cheung R, Perumpail RB, Harrison SA, Younossi ZM, Ahmed A. Nonalcoholic steatohepatitis is the second leading etiology of liver disease among adults awaiting liver transplantation in the United States. Gastroenterology. 2015;148(3):547-55.

[3] Younossi Z, Stepanova M, Ong JP, Jacobson IM, Bugianesi E, Duseja A, et al.; Global Nonalcoholic Steatohepatitis Council. Nonalcoholic steatohepatitis is the fastest growing cause of hepatocellular carcinoma in liver transplant candidates. Clin Gastroenterol Hepatol. 2019;17(4):748-755.e3. pii: S1542-3565(18)30611-6.

[4] Younossi ZM, Koenig AB, Abdelatif D, Fazel Y, Henry L, Wymer M. Global epidemiology of nonalcoholic fatty liver disease-meta-analytic assessment of prevalence, incidence, and outcomes. Hepatology. 2016;64(1):73-84. https://doi.org/10.1002/hep.28431.

[5] Lu, F.-B., Zheng, K. I., Rios, R. S., Targher, G., Byrne, C. D., and Zheng, M.-H. (2020) Global epidemiology of lean non-alcoholic fatty liver disease: A systematic review and meta-analysis. Journal of Gastroenterology and Hepatology, 35: 2041-2050 https://doi.org/10.1111/jgh.15156.

[6] Younossi ZM, Koenig AB, Abdelatif D, Fazel Y, Henry L, Wymer M. Global epidemiology of nonalcoholic fatty liver disease-meta-analytic assessment of prevalence, incidence, and outcomes. Hepatology. 2016;64(1):73-84. https://doi.org/10.1002/hep.28431.

[7] Ong JP, Elariny H, Collantes R, Younoszai A, Chandhoke V, Reines $\mathrm{HD}$, et al. Predictors of nonalcoholic steatohepatitis and advanced fibrosis in morbidly obese patients. Obes Surg. 2005;15(3):310-5

[8] Wong, Robert J., and Ramsey Cheung. "Trends in the Prevalence of Metabolic Dysfunction-Associated Fatty Liver Disease in the United States, 2011-2018." Clinical Gastroenterology and Hepatology (2021).

[9] Chen, Yu-ling, Hao Li, Shu Li, Zhou Xu, Shen Tian, Juan Wu, Xin-yu Liang et al. "Prevalence of and risk factors for metabolic associated fatty liver disease in an urban population in China: a cross-sectional comparative study." BMC gastroenterology 21, no. 1 (2021): 1-12.

[10] Estes C, Razavi H, Loomba R, Younossi Z, Sanyal AJ. Modeling the epidemic of nonalcoholic fatty liver disease demonstrates an exponential increase in burden of disease. Hepatology 2018;67:123133.

[11] Singh S, Allen AM, Wang Z, Prokop LJ, Murad MH, Loomba R. Fibrosis progression in nonalcoholic fatty liver vs nonalcoholic steatohepatitis: a systematic review and meta-analysis of paired-biopsy studies. Clin Gastroenterol Hepatol. 2015;13(4):643-54.e1-9.

[12] Patel YA, Gifford EJ, Glass LM, McNeil R, Turner MJ, Han B, et al. Risk factors for biopsy-proven advanced non-alcoholic fatty liver disease in the Veterans Health Administration. Aliment Pharmacol Ther. 2018;47(2):268-78. https://doi.org/10.1111/apt.14411.

[13] Sanyal, A.J.; Banas, C.; Sargeant, C.; Luketic, V.A.; Sterling, R.K.; Stravitz, R.T.; Shiffman, M.L.; Heuman, D.; Coterrell, A.; Fisher, R.A.; et al. Similarities and differences in outcomes of cirrhosis due to nonalcoholic steatohepatitis and hepatitis C. Hepatology 2006, 43, 682-689.

[14] McPherson S, Hardy T, Henderson E, Burt AD, Day CP, Anstee QM. Evidence of NAFLD progression from steatosis to fibrosingsteatohepatitis using paired biopsies: implications for prognosis and clinical management. J Hepatol. 2015;62(5):1148-55.

[15] Younossi ZM, Koenig AB, Abdelatif D, Fazel Y, Henry L, Wymer M. Global epidemiology of nonalcoholic fatty liver disease-meta-analytic assessment of prevalence, incidence, and outcomes. Hepatology. 2016;64(1):73-84. https://doi.org/10.1002/hep.28431.

[16] Pelusi S, Petta S, Rosso C, Borroni V, Fracanzani AL, Dongiovanni P, et al. Renin-angiotensin system inhibitors, type 2 diabetes and fibrosis progression: an observational study in patients with nonalcoholic fatty liver disease. PLoS One. 2016;11(9):e0163069.

[17] Porro, S.; Genchi, V.A.; Cignarelli, A.; Natalicchio, A.; Laviola, L.; Giorgino, F.; Perrini, S. Dysmetabolic adipose tissue in obesity: Morphological and functional characteristics of adipose stem cells and mature adipocytes in healthy and unhealthy obese subjects. $J$. Endocrinol. Investig. 2020, 1-21.
[18] Cimini, F.A.; Barchetta, I.; Ciccarelli, G.; Leonetti, F.; Silecchia, G.; Chiappetta, C.; Di Cristofano, C.; Capoccia, D.; Bertoccini, L.; Ceccarelli, V.; et al. Adipose tissue remodelling in obese subjects is a determinant of presence and severity of fatty liver disease. Diabetes Metab. Res. Rev. 2021, 37, e3358.

[19] Kim D, Kim WR. Nonobese fatty liver disease. Clin Gastroenterol Hepatol 2017;15:474-485.

[20] Liu, Jiaye, Ibrahim Ayada, Xiaofang Zhang, Ling Wang, Yang Li, Tianfu Wen, Zhongren Ma et al. "Estimating global prevalence of metabolic dysfunction-associated fatty liver disease in overweight or obese adults." Clinical Gastroenterology and Hepatology (2021).

[21] Kim D, Kim W, Joo SK, et al. Association between body sizemetabolic phenotype and nonalcoholic steatohepatitis and significant fibrosis. J Gastroenterol 2020;55:330-341.

[22] Davila, J.A. Diabetes and hepatocellular carcinoma: What role does diabetes have in the presence of other known risk factors? Am. $J$. Gastroenterol. 2010, 105, 632-634.

[23] Blond, E.; Disse, E.; Cuerq, C.; Drai, J.; Valette, P.-J.; Laville, M.; Thivolet, C.; Simon, C.; Caussy, C. EASL-EASD-EASO clinical practice guidelines for the management of non-alcoholic fatty liver disease in severely obese people: Do they lead to over-referral? Diabetologia 2017, 60, 1218-1222

[24] Lazo M, Hernaez R, Eberhardt MS, et al. Prevalence of nonalcoholic fatty liver disease in the United States: The Third National Health and Nutrition Examination Survey, 1988-1994. Am J Epidemiol 2013;178:38-45.

[25] Guerrero R, Vega GL, Grundy SM, Browning JD. Ethnic differences in hepatic steatosis: an insulin resistance paradox? Hepatology. 2009;49(3):791-801

[26] Romeo S, Kozlitina J, Xing C, Pertsemlidis A, Cox D, Pennacchio LA, et al. Genetic variation in PNPLA3 confers susceptibility to nonalcoholic fatty liver disease. Nat Genet. 2008;40:1461-5.

[27] Romeo S, Kozlitina J, Xing C, Pertsemlidis A, Cox D, Pennacchio LA, et al. Genetic variation in PNPLA3 confers susceptibility to nonalcoholic fatty liver disease. Nat Genet. 2008;40:1461-5.

[28] Mandorfer M, Scheiner B, Stattermayer AF, Schwabl P, Paternostro R, Bauer D, et al. Impact of patatin-like phospholipase domain containing 3 rs738409 G/G genotype on hepatic decompensation and mortality in patients with portal hypertension. Aliment Pharmacol Ther 2018;48:451.

[29] Liu YL, Patman GL, Leathart JB, Piguet AC, Burt AD, Dufour JF, et al. Carriage of the PNPLA3 rs738409 $\mathrm{C}>\mathrm{G}$ polymorphism confers an increased risk of non-alcoholic fatty liver disease associated hepatocellular carcinoma. J Hepatol. 2013;61(1):75-81.

[30] Sookoian, S.; Castaño, G.O.; Scian, R.; Mallardi, P.; Fernandez Gianotti, T.; Burgueño, A.L.; San Martino, J.; Pirola, C.J. Genetic variation in transmembrane 6 superfamily member 2 and the risk of nonalcoholic fatty liver disease and histological disease severity. Hepatology 2015, 61, 515-525.

[31] Mancina RM, Dongiovanni P, Petta S, Pingitore P, Meroni M, Rametta $\mathrm{R}$, et al. The MBOAT7-TMC4 variant rs641738 increases risk of nonalcoholic fatty liver disease in individuals of European descent. Gastroenterology. 2016;150(5):1219-30.e6.

[32] Santoro N, Zhang CK, Zhao H, Pakstis AJ, Kim G, Kursawe R, et al. Variant in the glucokinase regulatory protein (GCKR) gene is associated with fatty liver in obese children and adolescents. Hepatology. 2011;55:781-9.

[33] Pericleous M, Kelly C, Wang T, Livingstone C, Ala A. Wolman's disease and cholesteryl ester storage disorder: the phenotypic spectrum of lysosomal acid lipase deficiency. Lancet Gastroenterol Hepatol. 2017;2(9):670-9.

[34] Ma, Y.; Belyaeva, O.V.; Brown, P.M.; Fujita, K.; Valles, K.; Karki, S.; De Boer, Y.S.; Koh, C.; Chen, Y.; Du, X.; et al. 17-beta hydroxysteroid dehydrogenase 13 is a hepatic retinol dehydrogenase associated with histological features of nonalcoholic fatty liver disease. Hepatology 2019, 69, 1504-1519.

[35] Dongiovanni P, Meroni M, Mancina RM, Baselli G, Rametta R, Pelusi $S$, et al. Protein phosphatase 1 regulatory subunit $3 \mathrm{~B}$ gene variation protects against hepatic fat accumulation and fibrosis in individuals at high risk of nonalcoholic fatty liver disease. Hepatol Commun. 2018;2(6):666-75.

[36] Liu, Zhenqiu, Chen Suo, Oumin Shi, Chunqing Lin, Renjia Zhao, Huangbo Yuan, Li Jin, Tiejun Zhang, and Xingdong Chen. "The health impact of MAFLD, a novel disease cluster of NAFLD, is amplified by the integrated effect of fatty liver disease related genetic variants." Clinical Gastroenterology and Hepatology (2020).

[37] Caussy C, Soni M, Cui J, Bettencourt R, Schork N, Chen CH, et al. Nonalcoholic fatty liver disease with cirrhosis increases familial risk for advanced fibrosis. J Clin Invest. 2017;127(7):2697-704. 
[38] Breij LM, Kerkhof GF, Hokken-Koelega AC. Accelerated infant weight gain and risk for nonalcoholic fatty liver disease in early adulthood. J Clin Endocrinol Metab. 2014;99(4):1189-95.

[39] Valenti L, Romeo S. Destined to develop NAFLD? The predictors of fatty liver from birth to adulthood. J Hepatol. 2016;65(4):668-70.

[40] Gerhard GS, DiStefano JK. Micro RNAs in the development of nonalcoholic fatty liver disease. World J Hepatol. 2015;7(2):226-34.

[41] Kirpich IA, Marsano LS, McClain CJ. Gut-liver axis, nutrition, and non-alcoholic fatty liver disease. Clin Biochem 2015;48:923e930.

[42] Angulo P, Kleiner DE, Dam-Larsen S, Adams LA, Bjornsson ES, Charatcharoenwitthaya $\mathrm{P}$, et al. Liver fibrosis, but no other histologic features, is associated with long-term outcomes of patients with nonalcoholic fatty liver disease. Gastroenterology. 2015;149:389 397.e10. doi: 10.1053/j.gastro.2015.04.043.

[43] Dulai PS, Singh S, Patel J, et al. Increased risk of mortality by fibrosis stage in nonalcoholic fatty liver disease: systematic review and metaanalysis. Hepatology. 2017; 65: 1557- 1565.

[44] Nguyen, Vy H., Michael H. Le, Ramsey C. Cheung, and Mindie H. Nguyen. "Differential clinical characteristics and mortality outcomes in persons with NAFLD and/or MAFLD." Clinical Gastroenterology and Hepatology (2021).

[45] Stepanova M, Rafiq N, Makhlouf H, Agrawal R, Kaur I, Younoszai Z, McCullough A, Goodman Z, Younossi ZM. Predictors of all-cause mortality and liver-related mortality in patients with non-alcoholic fatty liver disease (NAFLD). Dig Dis Sci. 2013;58(10):3017-23.

[46] Mantovani, Alessandro, Graziana Petracca, Giorgia Beatrice, Alessandro Csermely, Amedeo Lonardo, Jörn M. Schattenberg, Herbert Tilg, Christopher D. Byrne, and Giovanni Targher. "Nonalcoholic fatty liver disease and risk of incident chronic kidney disease: an updated meta-analysis." Gut (2020).

[47] Lee, Hokyou, Yong-ho Lee, Seung Up Kim, and Hyeon Chang Kim. "Metabolic Dysfunction-Associated Fatty Liver Disease and Incident Cardiovascular Disease Risk: A Nationwide Cohort Study." Clinical Gastroenterology and Hepatology (2020).

[48] Deng, Yunlei, Qianwen Zhao, and Rong Gong. "Association Between Metabolic Associated Fatty Liver Disease and Chronic Kidney Disease: A Cross-Sectional Study from NHANES 2017-2018." Diabetes, Metabolic Syndrome and Obesity: Targets and Therapy 14 (2021): 1751.

[49] Sun, Dan-Qin, Yan Jin, Ting-Yao Wang, Kenneth I. Zheng, Rafael S. Rios, Hao-Yang Zhang, Giovanni Targher, Christopher D. Byrne, WeiJie Yuan, and Ming-Hua Zheng. "MAFLD and risk of CKD." Metabolism 115 (2021): 154433.

[50] Kim D, Li AA, Perumpail BJ, et al. Changing trends in etiology-based and ethnicity-based annual mortality rates of cirrhosis and hepatocellular carcinoma in the United States. Hepatology 2019;69:1064-1074.

[51] Vilar-Gomez E, Calzadilla-Bertot L, Wai-Sun Wong V, Castellanos M, Aller-de la Fuente R, Metwally M, et al. Fibrosis severity as a determinant of cause-specific mortality in patients with advanced nonalcoholic fatty liver disease: a multi-national cohort study. Gastroenterology. 2018;155(2):443-457.e17.

[52] Cholankeril G, Wong RJ, Hu M, Perumpail RB, Yoo ER, Puri P, et al. Liver transplantation for nonalcoholic steatohepatitis in the US: temporal trends and outcomes. Dig Dis Sci. 2017;62(10):2915-22.

[53] Wong RJ, Aguilar M, Cheung R, Perumpail RB, Harrison SA, Younossi ZM, Ahmed A. Nonalcoholic steatohepatitis is the second leading etiology of liver disease among adults awaiting liver transplantation in the United States. Gastroenterology. 2015;148(3):547-55.

[54] Nyberg, L.M., Cheetham, T.C., Patton, H.M., Yang, S.-J., Chiang, K.M., Caparosa, S.L., Stern, J.A. and Nyberg, A.H. (2021), The Natural History of NAFLD, a Community-Based Study at a Large Health Care Delivery System in the United States. Hepatol. Commun., 5: 83-96. https://doi.org/10.1002/hep4.1625.

[55] Myers, Shuna, Isabelle Neyroud-Caspar, Laurent Spahr, Konstantinos Gkouvatsos, Evelyne Fournier, Emiliano Giostra, Giulia Magini et al. "NAFLD and MAFLD as emerging causes of HCC: A populational study." JHEP Reports 3, no. 2 (2021): 100231.

[56] Kim D, Li AA, Perumpail BJ, et al. Changing trends in etiology-based and ethnicity-based annual mortality rates of cirrhosis and hepatocellular carcinoma in the United States. Hepatology 2019;69:1064-1074.

[57] Jafri, W., and M. Kamran. "NAFLD: The Enemy Within." Austin Hepatol 1.1 (2016): 1003.

[58] Srivastava, A.; Gailer, R.; Tanwar, S.; Trembling, P.; Parkes, J.; Rodger, A.; Suri, D.; Thorburn, D.; Sennett, K.; Morgan, S.; et al. Prospective evaluation of a primary care referral pathway for patients with non-alcoholic fatty liver disease. J. Hepatol. 2019, 71, 371-378.
[59] ${ }^{1}$ de Freitas Junior, J. R., V. M. T. Sagae, G. H. P. de Oliveira, G. M V. de Souza, T. F. de Souza, C. P. M. S. de Oliveira, D. T. H. de Moura et al. "Intragastric Balloon as a New Approach for Metabolic Dysfunction-Associated Fatty Liver Disease (Mafld): A Systematic Review and Meta-Analysis." Endoscopy 53, no. S 01 (2021): OP42.

[60] Vilar-Gomez, E.; Martinez-Perez, Y.; Calzadilla-Bertot, L.; TorresGonzalez, A.; Gra-Oramas, B.; Gonzalez-Fabian, L.; Friedman, S.L.; Diago, M.; Romero-Gomez, M. Weight loss through lifestyle modification significantly reduces features of nonalcoholic steatohepatitis. Gastroenterology 2015, 149, 367-378.e5.

[61] Wong VW, Wong GL, Choi PC, et al. Disease progression of nonalcoholic fatty liver disease: a prospective study with paired liver biopsies at 3 years. Gut. 2010; 59: 969- 974.

[62] T. Andersen, C. Gluud, M.-B. Franzmann, and P. Christoffersen, "Hepatic effects of dietary weight loss in morbidly obese subjects," Journal of Hepatology, vol. 12, no. 2, pp. 224-229, 1991.

[63] Hamurcu Varol P, Kaya E, et al. Role of intensive dietary and lifestyle interventions in the treatment of lean nonalcoholic fatty liver disease patients. Eur J Gastroenterol Hepatol, 2020.

[64] M Zachut, A Arieli, H Lehrer, L Livshitz, S Yakoby, U Moallem: Effects of increased supplementation of $n-3$ fatty acids to transition dairy cows on performance and fatty acid profile in plasma, adipose tissue, and milk fat. J Dairy Sci 93, 5877-5889 (2010).

[65] H Yki-Järvinen: Nutritional modulation of nonalcoholic fatty liver disease and insulin resistance: human data. Curr Opin Clin Nutr Metab Care (2010).

[66] Ryan MC, Itsiopoulos C, Thodis $\mathrm{T}$, et al. The Mediterranean diet improves hepatic steatosis and insulin sensitivity in individuals with non-alcoholic fatty liver disease. $J$ Hepatol 2013;59:138-43. 10.1016/j.jhep.2013.02.012.

[67] J Frith, CP Day, L Robinson, C Elliott, DE Jones, JL Newton: Potential strategies to improve uptake of exercise interventions in non-alcoholic fatty liver disease. J Hepatol 52, :112-116 (2010).

[68] O’Gorman, Philip, Sara Naimimohasses, Ann Monaghan, Megan Kennedy, Ashanty M. Melo, Deirdre Ní Fhloinn, Derek G. Doherty et al. "Improvement in histological endpoints of MAFLD following a 12week aerobic exercise intervention." Alimentary Pharmacology \& Therapeutics 52, no. 8 (2020): 1387-1398.

[69] KD Kistler, EM Brunt, JM Clark, AM Diehl, JF Sallis, JB Schwimmer: Physical activity recommendations, exercise intensity, and histological severity of nonalcoholic Fatty liver disease. Am J Gastroenterol 106, 460-468 (2011).

[70] Chalasani N, Younossi Z, Lavine JE, et al. The diagnosis and management of nonalcoholic fatty liver disease: Practice guidance from the American Association for the Study of Liver Diseases. Hepatology 2018;67:328e357.

[71] EASL-EASD-EASO Clinical Practice Guidelines for the management of non-alcoholic fatty liver disease. J Hepatol 2016;64:1388e1402.

[72] Venetsanaki V, Karabouta Z, Polyzos SA. Farnesoid X nuclear receptor agonists for the treatment of nonalcoholic steatohepatitis. Eur J Pharmacol 2019;863:172661.

[73] Niriella MA, Ediriweera DS, Kasturiratne A, De Silva ST, Dassanayaka AS, De Silva AP, Kato N, Pathmeswaran A, Wickramasinghe AR, de Silva HJ. Outcomes of NAFLD and MAFLD: Results from a community-based, prospective cohort study. PLoS One. 2021 Feb 3;16(2):e0245762. doi: 10.1371/journal.pone.0245762. 